\title{
PLANEJAMENTO ESTRATÉGICO SETORIAL 2025 - ROADMAP IoT
}

Paulo Vítor Guerra (paulo.guerra@iebt.com.br) - IEBT

Bruno França Pádua Coelho (bruno.padua@ iebt.com.br) - IEBT

Bruna Silva Barbosa Pereira (bruna.pereira@ iebt.com.br) - IEBT

Maicon Gouvêa de Oliveira (maicon.oliveira@unifal-mg.edu.br) - Instituto de Ciência e Tecnologia, Universidade Federal de Alfenas

Isadora Giannetti Viotti (isadora.viotti@iebt.com.br) - IEBT

\section{RESUMO}

O método do Roadmapping foi utilizado para planejamento estratégico de setores industriais mineiros no âmbito de um projeto desenvolvido em parceria com o Serviço Brasileiro de Apoio às Micro e Pequenas Empresas de Minas Gerais (SEBRAE-MG), cujo tema central foi o setor de Internet da Coisas (IoT). Os objetivos do estudo foram a identificação de oportunidades de atuação para as micro e pequenas empresas e a proposição de uma agenda de atuação conjunta entre diferentes atores na realização de ações estratégicas que viabilizassem a implementação da IoT de forma alinhada às dificuldades reais relatadas por empresas e empreendedores; possibilitando assim, a consolidação e a competitividade das empresas nacionais e mineiras, com alcance internacional.

Para tanto, foi realizado diagnóstico do estado atual e proposta uma visão de futuro para o desenvolvimento de negócios e tecnologias no campo. Aplicou-se a metodologia Roadmapping para delinear rotas estratégicas até 2025. Os mapas foram gerados a partir de análises de mercado, estruturação da cadeia de valor da IoT e entrevistas com representantes de empresas e especialistas. Em seguida foi traçado um panorama das potencialidades de evolução da área de IoT, seus principais domínios de aplicação, os desafios que devem ser superados e os caminhos a serem trilhados para seu desenvolvimento no Brasil.

Como resultado foram mapeados 18 cenários para implementação da IoT, agrupados em seis domínios: pessoal, residencial, veicular, empresarial, industrial e espaços públicos. O desenho das rotas estratégicas também serviu como base para o direcionamento de ações, por meio da identificação de gaps e seleção dos principais recursos necessários para construção do futuro almejado em cada tema estratégico. Foram propostas ações estratégicas distribuídas em sete categorias: governamental e regulatório; humanos, formação; tecnológicos; infraestrutura; financeiros; sociais; e, gerenciais.

Palavras-chave: Strategic Roadmapping, Internet das Coisas, Planejamento Estratégico Setorial Área: Potencial da Internet of Things (IoT) e soluções de TIC para o desenvolvimento de produtos e serviços

\section{INTRODUÇÃO}

O Serviço Brasileiro de Apoio às Micro e Pequenas Empresas de Minas Gerais - SEBRAEMG viabilizou a realização de um planejamento estratégico para os setores de energia elétrica 
e internet das coisas (IoT). O objetivo foi além da identificação de oportunidades de atuação para as micro e pequenas empresas (MPEs) do estado de Minas Gerais, passando por formas de apoiá-las a alcançar novos mercados, produtos de alto valor agregado e tecnologias. Foram identificadas oportunidades de atuação conjunta entre diferentes agentes na realização de ações estratégicas que viabilizem a implementação da Internet das Coisas de forma alinhada às dificuldades relatadas por empresas e empreendedores, possibilitando assim, a consolidação e competitividade das empresas nacionais e mineiras.

Aplicou-se a metodologia Roadmapping, difundida mundialmente por pesquisadores da Universidade de Cambridge, para delinear rotas estratégicas para os próximos 10 anos. Tais rotas foram construídas a partir do entendimento da dinâmica e tendências de mercado, análise da cadeia de valor, demandas dos negócios, novidades em produtos, tecnologias e serviços, inseridos no contexto econômico e social do país. Assim, foi traçado um panorama das potencialidades de evolução da área de Internet das Coisas, seus principais domínios de aplicação, os desafios que devem ser superados e os caminhos a serem trilhados para seu desenvolvimento no Brasil.

A consolidação da IoT no Brasil dependerá de mudanças que vão além das tecnologias emergentes. A atuação conjunta e coerente dos agentes nacionais e empreendedores é um esforço chave para o sucesso na aplicação das tecnologias em serviços relevantes. $O$ planejamento do setor de forma colaborativa é um primeiro passo para impulsionar a área, gerando impacto econômico a partir do aproveitamento de oportunidades de mercado para as micro e pequenas empresas.

Os mapas foram gerados a partir de análises de mercado, estruturação da cadeia de valor da IoT, entrevistas com representantes de empresas e especialistas. O desenho das rotas estratégicas serviu como base para o direcionamento de ações convergentes entre os agentes do setor, por meio da identificação de gaps e seleção dos principais recursos necessários para construção do futuro almejado em cada tema estratégico.

Os principais desafios ao desenvolvimento da IoT no Brasil foram identificados e distribuídos em seis temas: Design, Conectividade, Interoperabilidade, Segurança e Privacidade, Gestão e Qualidade, e Orientação à Serviço. Os mesmos deram origem à proposição de 58 ações estratégicas, distribuídas em sete categorias: governamental e regulatório; humanos; tecnológicos; infraestrutura; financeiros; sociais; e gerenciais.

\section{ROADMAPPING COMO FERRAMENTA PARA PLANEJAMENTO ESTRATÉGICO DE MÚLTIPLOS SETORES}

O método do Roadmapping, desenvolvido pelos pesquisadores do IfM (Institute for Manufacturing) da Universidade de Cambridge no Reino Unido, configura-se no processo de construção e representação gráfica da rota de evolução do mercado, produtos e tecnologias, alinhando as ações de desenvolvimento com as metas almejadas (PHAAL, FARRUKH e PROBERT, 2010 e OLIVEIRA et al. 2012).

O roadmapping é uma abordagem utilizada para a identificação, definição e mapeamento de estratégias, objetivos e ações relacionados com a inovação, sendo seu principal resultado o roadmap, um mapa que integra perspectivas de áreas distintas com o objetivo de alinhar diferentes visões para responder de forma coordenada a três perguntas "onde estamos", "aonde queremos chegar" e "como chegaremos lá" (OLIVEIRA et. al., 2012). O método foi utilizado para dar suporte ao planejamento setorial de longo prazo do setor de Internet das Coisas, comunicando graficamente as mudanças, tendências, e influências internas e externas, bem como seus impactos, e fazendo a ligação entre as oportunidades de mercado, produto e o 
desenvolvimento das tecnologias. Os principais objetivos do mapa são facilitar a comunicação, a colaboração e a tomada de decisão compartilhada, mediante a definição de recursos e programas de apoio necessários para consolidação de cada rota identificada, sendo sua característica essencial o uso de uma estrutura gráfica que "conta", visualmente, uma história fornecendo uma linguagem comum aos envolvidos, explorando as relações entre múltiplas camadas interdependentes ao longo do tempo.

Segundo Phaal, Farrukh e Probert (2010), o desafio para a gestão da inovação e tecnologia é o desenvolvimento de um processo efetivo e um sistema para garantir que os investimentos tecnológicos estão alinhados às necessidades atuais e futuras do mercado. O impacto das mudanças tecnológicas e das necessidades do mercado precisam ser entendidas, em termos das potenciais ameaças e oportunidades. Esses temas vão ficando mais críticos com o aumento dos custos, complexidade e velocidade de mudanças tecnológicas, bem como a competividade e recursos tecnológicos globalizados, situações comuns quando o tema é Internet das Coisas. A gestão estratégica da tecnologia é desafiadora, devido à complexidade dos sistemas, às incertezas relacionadas aos mercados futuros e aos desenvolvimentos tecnológicos, e à necessidade de alinhar o pensamento e a ação entre as funções dentro do negócio e entre as organizações envolvidas no sistema de inovação.

Devido a isso, o interesse no roadmapping tem crescido substancialmente nas últimas décadas, tanto em empresas como nos governos, conforme Figura 1. Isso foi permitido pela natureza flexível da abordagem que pode ser adaptada para abordar uma ampla gama de objetivos, sua capacidade de atuar como um mecanismo de integração para lidar com questões estratégicas complexas e incertas, foco na tomada de decisões e na ação, e talvez, mais importante seja o poder de apoiar a comunicação e a construção consensual.

Figura 1 - Evolução da utilização do roadmapping.

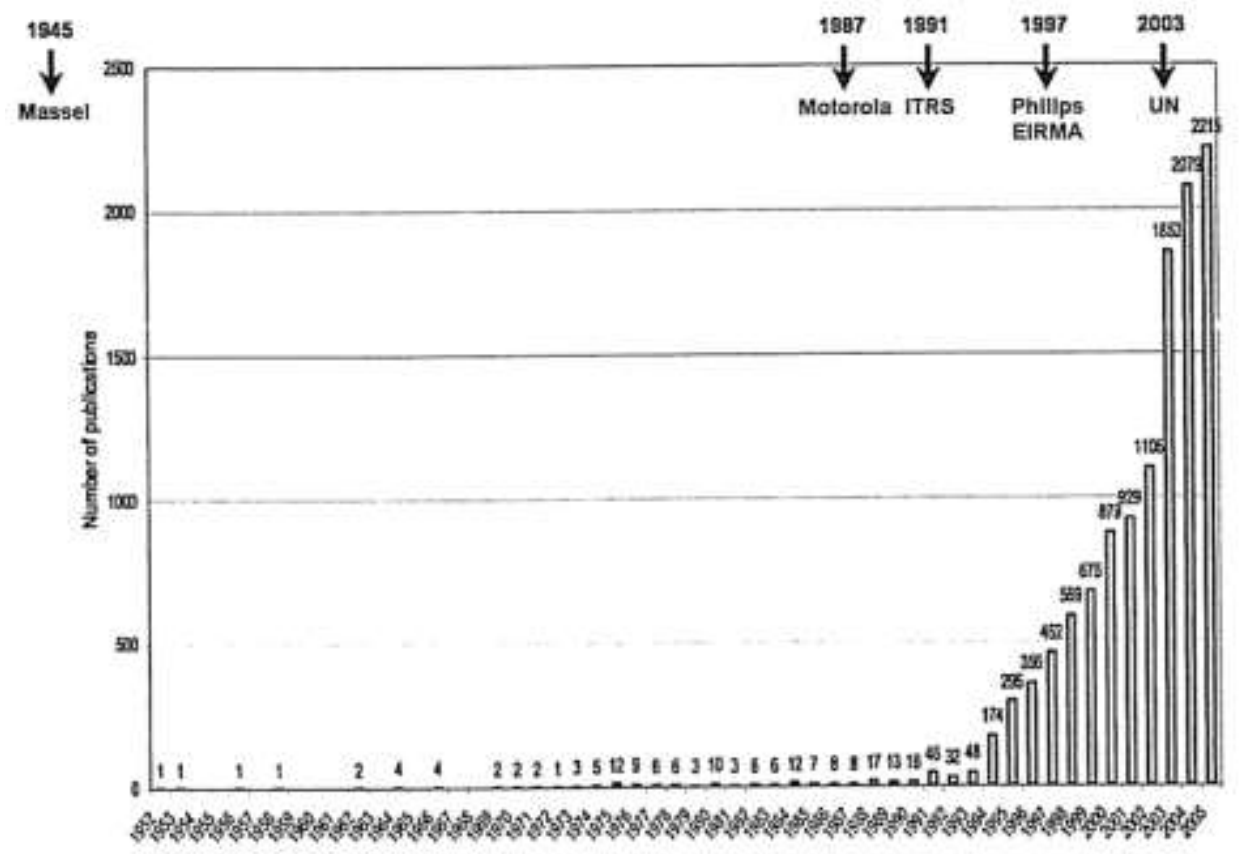

Fonte: Phaal, Farrukh e Probert (2010)

Por causa da grande variedade de aplicações potenciais, que variam em âmbito de produtos individuais a setores inteiros, a estrutura do roadmap e seu processo de construção dependerão 
da perspectiva tomada e do propósito da iniciativa que podem ser tecnológicas, inovação, estratégica ou setor industrial. A perspectiva adotada no presente trabalho foi setorial.

Ainda segundo os autores, as organizações não operam de forma isolada, mas fazem parte de um sistema dinâmico maior, que inclui seus clientes, concorrentes, fornecedores e órgãos governamentais. As empresas também não evoluem isoladamente, uma vez que tecnologias desenvolvidas por uma impactam diretamente em outras. Assim, se uma organização deve desenvolver uma estratégica eficaz para se orientar, contando com produtos e serviços que proporcionem sucesso sustentável, é importante que ela entenda o sistema mais amplo no qual opera. Isso requer consciência das tendências e direcionadores externos, sociais, econômicos, ambientais, industriais, tecnológicos e políticos, e como eles podem interagir e evoluir. Os mesmos fatores são de interesse para investidores, agências de financiamento e departamentos governamentais, que precisam tomar decisões de investimento e políticas, tendo uma perspectiva mais ampla do que empresas individuais.

Nesse sentido, as camadas superiores do mapa devem mostrar claramente os fatores externos que influenciam o negócio, que operam em diferentes níveis:

a) Tendências e direcionadores de nível macro, que influenciam a maioria das indústrias, como sociais, tecnológicos, econômicos, ambientais, políticos, legais e fatores de infraestrutura.

b) Drivers específicos da indústria, como o impacto da regulamentação e padrões, o comportamento dos concorrentes e a dinâmica da cadeia de suprimentos.

c) Tendências, necessidades e requisitos do cliente, que influenciam diretamente as decisões de compra.

Figura 2 - Modelo conceitual do roadmap.

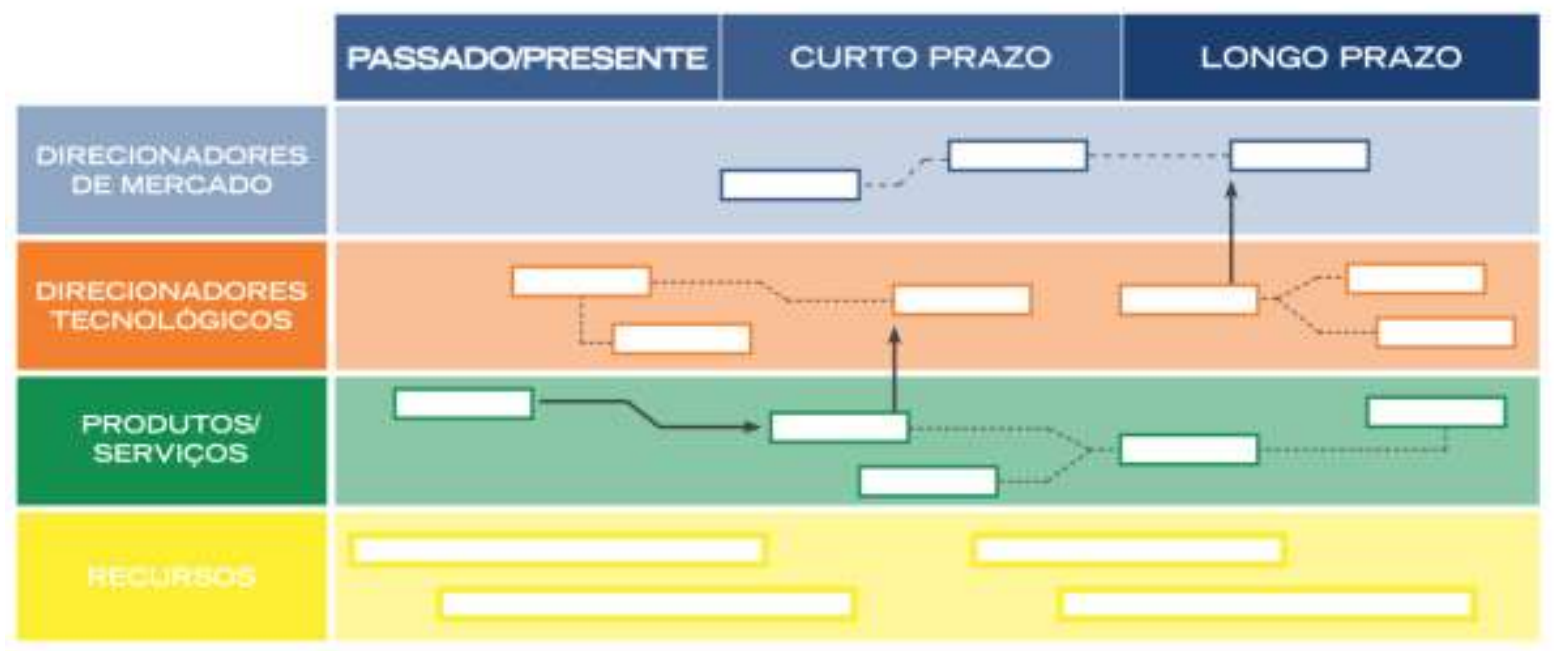

Fonte: adaptado de Phaal e Probert (2004)

No nível setorial, as organizações evoluem em resposta às forças que as influenciam. Este ecossistema setorial é complexo e adaptativo, com muitas organizações em todos os níveis tomando decisões e agindo com base na melhor compreensão do sistema, do futuro, e das opções disponíveis para eles. As indústrias emergem, crescem, amadurecem e diminuem, com organizações e populações sujeitas a mecanismos de seleção evolutiva impostas principalmente por meio de forças competitivas. 


\section{INTERNET DAS COISAS COMO ELEMENTO DE COMPETIVIDADE DE MÚLTIPLAS INDÚSTRIAS}

A Internet das Coisas (em inglês, Internet of Things - IoT) pode ser definida de diferentes formas, por diversos atores. De acordo com a Juniper Research (2016) IoT é "a combinação de dispositivos e sistemas de software, ligados por meio da internet, que produzem, recebem e analisam dados. Devem ter o objetivo de transcender ecossistemas tradicionais de informação eletrônica, a fim de melhorar a qualidade de vida, eficiência, criar valor e reduzir custos". Também pode ser entendido como "coisas" habilitadas com conexão plena, capazes de gerar dados que podem ser analisados para extrair informações valiosas (INTEL, 2014).

No presente trabalho adota-se a definição de IoT como um conjunto de redes interligadas formadas por pessoas, objetos e lugares plenamente conectados, identificados individualmente, monitorados e controlados remotamente em tempo real, contando com recursos de segurança e opções de privacidade sob controle do usuário. A interação com dispositivos e aplicações de forma mais fluida e humanizada possível promoverá engajamento contínuo com as soluções e personalização pelo usuário, uma aproximação entre empresa e cliente, que permitirá direcionar a criação de valor da tecnologia e usabilidade cada vez mais justa com as necessidades e comportamentos de diferentes perfis de usuários. A ampliação do uso de soluções abertas, melhor integração entre as empresas e cultura de compartilhamento de recursos irão ampliar a capacidade de inovação e demonstração de valor, exigindo menor investimento.

De forma sumarizada, a IoT é a interconexão em rede entre objetos cotidianos dotados das capacidades de (i) identificação única: endereçar individualmente as coisas; (ii) sensoriamento: transformar um fenômeno físico em dados digitais; (iii) conectividade: comunicar com outros objetos ou com uma rede; (iv) processamento: analisar e tomar decisões; (v) atuação: desempenhar uma função local.

O fato é que a habilidade de monitorar e controlar as coisas no mundo físico de forma digital tem inspirado uma onda de inovação e entusiasmo. Os próximos anos irão testemunhar a geração de um imensurável fluxo de dados à medida que praticamente tudo poderá ser instrumentalizado e sensorizado. A Internet das Coisas vai combinar este volume de dados com as últimas inovações em inteligência artificial, aprendizado de máquina, visualização de dados e aplicar este conhecimento para tomar decisões mais inteligentes, enxergar o futuro de forma mais clara, eliminar ineficiências e entender melhor os consumidores.

A evolução da IoT acompanha a evolução da própria internet, que passou por três principais eras, conectando primeiramente lugares, posteriormente pessoas e por fim objetos, ou seja, a Internet das Coisas. Esse crescimento é ilustrado pelo número de conexões geradas no passado e potencialmente no futuro. Em 1975, um bilhão de lugares estavam conectados; já no ano 2000 mais de 5 bilhões de pessoas estavam conectadas; e, em 2020 projeta-se mais de 50 bilhões de dispositivos conectados. Hoje a IoT é possível graças aos avanços tecnológicos ocorridos nos últimos anos, porém esse processo de evolução ainda está em curso, havendo ainda desafios que precisam ser superados para expansão das aplicações de IoT e sua adoção em larga escala.

A extensão das mudanças que a Internet das Coisas poderá trazer à gestão de empresas, negócios, recursos naturais e cidades, à forma que as pessoas monitoram sua saúde e estilo de vida, entre outras, ainda é obscura e tem gerado discussões e visões de futuro diversas. Entretanto, essas mudanças vêm se tornando realidade rapidamente, clamando por ações estruturadas e coordenadas entre empresas, governos e sociedade para que os benefícios trazidos pela IoT possam ser percebidos e aproveitados por todos. Segundo estudos da Gartner (2014) a IoT ocupava em 2014 o mais alto nível de expectativas de impacto nos setores industriais com o pico nos próximos 5 a 10 anos, conforme Figura 3. 
Figura 3 - $O$ ciclo das tecnologias emergentes.

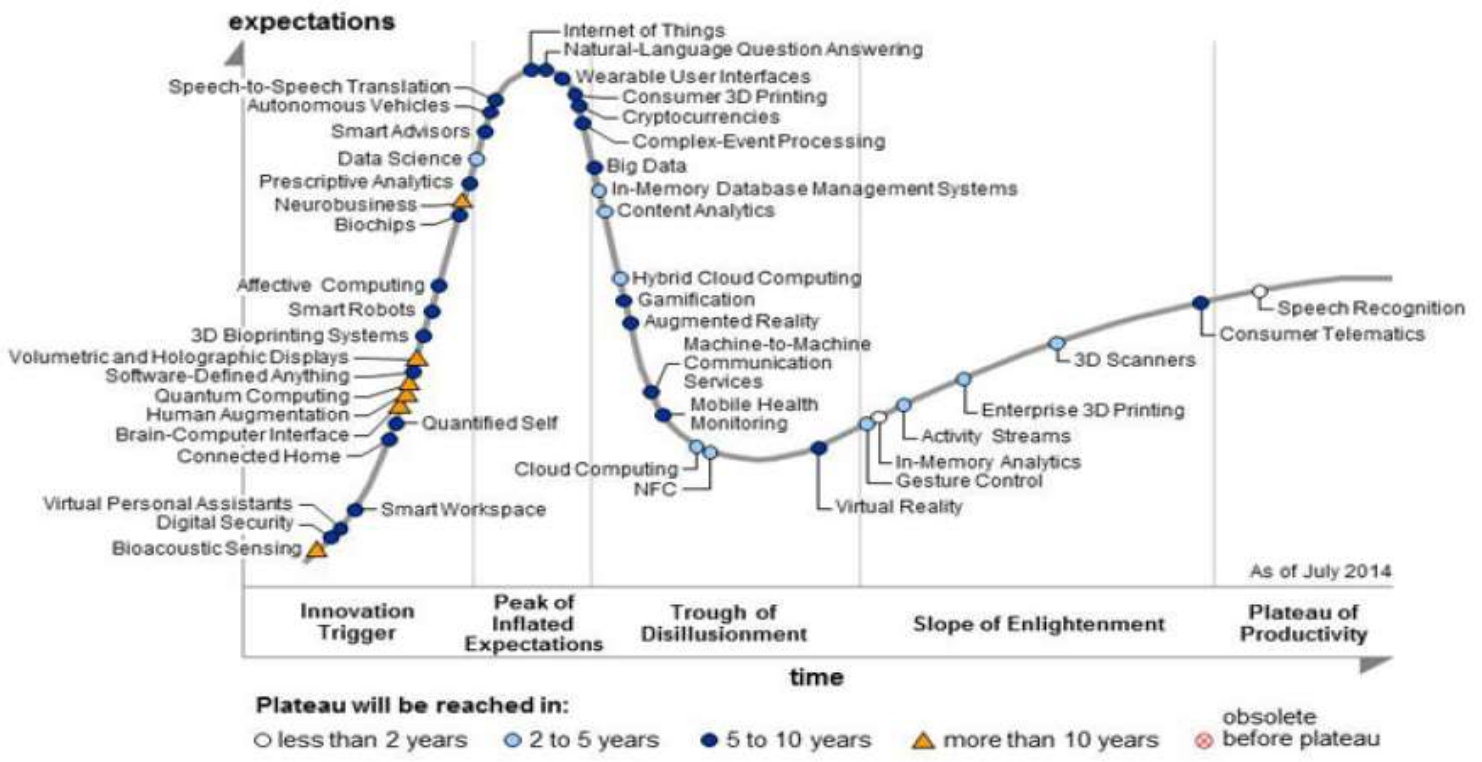

Fonte: Gartner (2014)

A expectativa, conforme apresentado por Gartner (2014), se deve ao fato de que a IoT irá permitir maior conhecimento sobre o mundo que nos rodeia e, como o advento da própria internet, possibilitará o surgimento de um vasto número de aplicações, das triviais às mais críticas. Seu papel não se restringe a tornar a vida das pessoas mais conveniente, mas seu maior valor será como ferramenta para gerir recursos de forma colaborativa e integrada, do indivíduo para uma escala global. Esta promessa de futuro depende de uma profunda reestruturação econômica, na forma como as organizações se governam e como interagimos com a tecnologia, para que se explore todo o leque de oportunidades oferecido para atuação de novos entrantes e o surgimento de produtos e serviços inovadores.

O sucesso dos serviços na área da Internet das Coisas demanda o estabelecimento de uma estrutura condizente com as necessidades de comunicação, integração, agilidade e baixo custo no desenvolvimento de soluções. Atualmente, a debilidade da matriz de telecomunicações brasileira, a falta de maturidade na adoção de técnicas de segurança e baixa preocupação com este aspecto no desenvolvimento das soluções, são exemplos de barreiras para adoção em larga escala que podem retrair a adesão às soluções e encontrar resistência do mercado. O crescente número de dispositivos conectados e o volume de dados gerados representam um desafio de gerenciamento. As empresas carecem de direcionamento estratégico e aproximação para sólidas relações de parceria com outras empresas atuantes na área.

\section{PROCESSO DE PLANEJAMENTO SETORIAL UTILIZANDO O ROADMAPPING}

O método do Roadmapping, desenvolvido pelos pesquisadores do IfM (Institute for Manufacturing) da Universidade de Cambridge, configura-se no processo de construção e representação gráfica da rota de evolução do mercado, produtos e tecnologias, alinhando às ações de desenvolvimento com as metas almejadas (PHAAL e PROBERT, 20004). O principal guia para aplicação utilizado no presente trabalho foi apresentado por Oliveira et. al. (2012), na explanação do Roadmapping do S-Plan (strategic plan). Entretanto, como os próprios autores destacam o processo é flexível e pode ser adaptado para diferentes contextos. 
Segundo os autores, o S-Plan é um processo de roadmapping criado para uso em unidades de análise de grande abrangência, visto que é capaz de considerar muitos tipos de informações e longos horizontes de tempo. Dessa forma, ele propicia a identificação, priorização e exploração de questões críticas para a definição das estratégias de inovação. Seu processo padrão está baseado em workshops divididos em três etapas: mapeamento estratégico, detalhamento de tópicos e revisão de resultados. É precedido pelo planejamento do processo e seguido pela implementação dos resultados.

$\mathrm{Na}$ atividade de planejamento são definidos os participantes, o foco de aplicação, as adaptações do processo padrão e a necessidade de trabalho preparatório para o workshop. Na etapa de mapeamento estratégico a arquitetura do roadmap é utilizada para capturar as visões dos participantes sobre a unidade de análise e para identificar, agrupar e priorizar os tópicos estratégicos. No detalhamento dos tópicos, aqueles priorizados anteriormente são explorados por pequenos grupos usando-se uma arquitetura simplificada de roadmap, que deve originar propostas de objetivos e ações. Na etapa de revisão, os roadmaps são apresentados para discussão envolvendo todos os grupos. Então, as propostas criadas são revisadas e consolidadas.

No presente estudo, desenvolvido durantes oito meses, o workshop de aplicação do roadmapping foi apenas a terceira de quatro etapas. A primeira delas envolveu a análise e entendimento da dinâmica do setor de IoT, por meio de pesquisa em materiais de referência e construção da cadeia de valor, identificando os principais players de cada elo. A segunda referiu-se ao levantamento de dados primários sobre o setor de IoT, tendo em vista a identificação de tendências, oportunidades, desafios e gaps de mercado. Ao final da etapa, buscou-se a construção da versão preliminar do roadmap setorial. A terceira etapa englobou a realização de dinâmicas de construção colaborativa, para complementar e validar os dados levantados, como forma de fomentar o planejamento da rota estratégica do setor, conforme orientação do S-Plan. Ao final, teve-se a elaboração da versão final do mapa estratégico. Por fim, na quarta etapa seguiu-se com o estabelecimento de um plano de ações que serviria de base para a construção de uma agenda convergente de investimentos e incentivos no setor entre agentes como Governo de Minas, SEBRAE-MG, Federação de Indústrias, setor privado e entidades de fomento.

\section{ROTAS E PLANO DE AÇÃO ESTRATÉGICO PARA O SETOR}

Desenvolvido durante oito meses, o projeto contou com 87 participantes de 58 instituições, gerando um conjunto de 58 ações estratégicas para promoção da IoT e aproveitamento de oportunidades de negócios para empresas brasileiras e mineiras. Foram realizadas duas dinâmicas de construção colaborativa, com a participação de 21 convidados, que contribuíram para identificação e validação das tendências e demandas nacionais e internacionais, atuais e futuras, e os recursos necessários para estas diretrizes foram endossados. Foram mapeados 18 cenários para implementação da IoT, agrupadas em seis domínios de aplicações. São eles:

- Pessoal: as informações geradas pelas soluções de IoT permitirão aprender sobre as preferências do usuário, responder às suas necessidades, conhecer seu contexto e forma de uso e emitir feedbacks. Os principais benefícios serão voltados para saúde, prática de atividades físicas, esportes e bem-estar. A coleta de dados pessoais permitirá a formação de um histórico e perfil individual, guiando a ação e tomada de decisão dos usuários e dos profissionais da área da saúde e aconselhamento pessoal. Soluções integradas irão compartilhar informações em tempo real, oferecendo novas formas de entretenimento e comunicação.

- Residencial: residências e conjuntos habitacionais serão transformados pela capacidade de monitorar, automatizar, controlar remotamente e integrar o ambiente. As aplicações 
nesse domínio irão poupar tempo na execução de tarefas domésticas, oferecer maior comodidade e segurança aos moradores e permitir novas formas de interação, personalização e contextualização. Informações precisas sobre o padrão de consumo de utilidades irão facilitar seu controle, permitindo maior ciência no uso desses recursos e influenciando melhorias nos hábitos de consumo.

- Veicular: as aplicações no domínio veicular irão conectar carros, motoristas, fabricantes, prestadores de serviços, seguradoras e infraestrutura viária. Segundo estimativas, o número de carros conectados no mundo crescerá de 26 milhões para 152 milhões até 2020. O compartilhamento de informações e emissão de dados em tempo real irá permitir novas formas de entretenimento e integração, alinhando as preferências do usuário e comunicando com diversos serviços. O uso massivo de sensores permitirá a automação de funções, conferindo autonomia total ou parcial aos veículos.

- Empresarial: soluções no domínio empresarial serão beneficiadas pela capacidade de identificar, monitorar, compartilhar, analisar informações, interagir remotamente e rastrear indivíduos e ativos. O compartilhamento de dados irá aproximar as empresas de seus consumidores e integrar os diversos stakeholders, tornando-os mais eficientes e produtivos. Informações precisas sobre os consumidores irão permitir uma melhor segmentação de clientes e a customização de produtos, serviços e ofertas. O surgimento de novas formas de pagamento, integradas com os dispositivos pessoais dos consumidores, irá trazer maior segurança e comodidade. A capacidade de integrar dispositivos e sistemas irão moldar os espaços de trabalho e aprendizado, facilitando o treinamento e integração de equipes, inclusive remotamente.

- Industrial: as aplicações da IoT irão transformar completamente os ambientes de produção, podendo representar a próxima revolução industrial, a Indústria 4.0. A capacidade de analisar grandes volumes de dados em tempo real irá aumentar os níveis de utilização e flexibilidade das plantas industriais, melhorar o diagnóstico de falhas em equipamentos e a eficiência na identificação de defeitos. A integração com sistemas de fornecedores, parceiros e clientes irá permitir um maior conhecimento sobre seus interesses e características, sendo úteis para o redesenho dos processos, produtos e serviços prestados. Áreas produtivas que oferecem risco para a saúde humana poderão ser monitoradas, evitando acidentes, e a robótica poderá ser utilizada para reduzir a necessidade de presença humana.

- Espaços Públicos: as aplicações nos espaços públicos irão reduzir gastos e gerar novas fontes de receita a governos e instituições públicas, como resultado da maior eficiência na distribuição e gestão de utilidades - água, energia - do lixo urbano, do trânsito e da infraestrutura e ativos. Os cidadãos terão melhores experiências na utilização de serviços públicos, como no acesso aos sistemas públicos de transporte com informações em tempo real, e a interação com os entes públicos será facilitada. A utilização de sensores irá aumentar a qualidade e efetividade dos serviços de emergência, segurança e ambientais. A prevenção e investigação de crimes e violência serão mais efetivas e fenômenos naturais serão previstos com maior precisão, sendo também possível monitorar áreas protegidas ou em risco, especialmente em locais remotos e de difícil acesso.

Cada um dos seis temas é contextualizado com um diagnóstico das condições atuais - estado da arte - em contraste com a visão do que se deseja alcançar - visão de futuro, ilustrado com dados e gráficos. O caminho que interliga o estado atual com a visão de futuro é traçado no roadmap, que consiste em uma projeção na linha do tempo de rotas estratégicas, isto é, os recursos necessários para desenvolvimento de soluções em produtos e serviços voltados para demandas e direcionadores de mercado e negócios. As rotas estratégicas são representadas por 
cores nos roadmaps, com a função de conectar as três camadas estruturais: direcionadores de mercado; soluções em produtos, serviços e tecnologias; e recursos, todas posicionadas na linha do tempo.

- Direcionadores de mercado: apresenta as necessidades dos usuários e pequenas e médias empresas da área. Também contempla tendências relacionadas ao tema e condições socioeconômicas, legislativas e de governança.

- Soluções em produtos, serviços e tecnologias: reúne soluções direcionadas aos aspectos abordados na camada dos direcionadores de mercado. Sugere-se soluções para transpor os desafios e alcançar as diretrizes de mercado que já estão disponíveis para ser adotadas, ou desejadas, como oportunidades de inovação. Podem ser plataformas, aplicações, protocolos, serviços, produtos, ou seja, engloba tecnologias e soluções pertencentes aos diversos elos da cadeia de valor da IoT.

- Recursos: identifica os recursos necessários para que as tendências que irão suportar este cenário e as necessidades identificadas no mercado sejam atendidas e se concretizem. Os recursos também podem servir às soluções, dando suporte para que estejam disponíveis e sejam adotadas.

O plano de ações convergentes resultou em 58 ações mapeadas, agrupadas em sete temas: (i) governamental e regulatório: adequações na legislação e governança; (ii) humanos e formação: capacitação e conscientização dos profissionais e usuários das aplicações; (iii) tecnológicos: demandas por pesquisa e desenvolvimento ou maior adoção de soluções necessárias à evolução da IoT; (iv) infraestrutura: criação e aperfeiçoamento dos ativos de rede e ambientes de inovação; (v) financeiros: atração e alocação dos recursos necessários; (vi) sociais: articulação de parcerias; e (vii) gerenciais: melhoria da performance dos negócios relacionados à IoT.

\section{CONCLUSÕES}

É evidente o enorme potencial da Internet das Coisas em promover uma profunda mudança na forma como entendemos e interagimos com o mundo. Benefícios a muito tempo almejados poderão ser observados em um novo patamar: aumento de produtividade, eficiência na gestão de recursos, redução de custos de criação de soluções com maior assertividade, melhorias na qualidade de vida e conveniência, acesso à informação e a serviços de primeira necessidade, entre outros, com impacto no crescimento econômico. Para a realização desta expectativa, é essencial a atuação conjunta dos agentes nacionais e empreendedores e a promoção de medidas adicionais para evolução do cenário nacional atual para pleno aproveitamento das oportunidades geradas pela IoT.

$\mathrm{O}$ advento da IoT abrirá um leque de oportunidades de mercado para empresas dos mais variados portes e áreas de atividade para ocupar um vasto espaço para criação de soluções inovadoras em produtos e serviços. Apesar dos diversos e profundos desafios explorados neste estudo, a Internet das Coisas é um caminho sem volta. Por isto é tão importante a mobilização para estruturação em sólidas bases dos variados setores envolvidos, para que as soluções sejam bem-sucedidas em suas propostas e adotadas em larga escala.

A metodologia do roadmapping apresentou-se robusta o suficiente para auxiliar no planejamento de ambientes complexos como o da IoT. A construção de uma visão de futuro de forma colaborativa, seja esta obtida por meio das entrevistas em campo (etapa 2) ou consolidado nos workshops (etapa 3) foi essencial para estabelecimento e entendimento dos desafios do setor, bem como elaboração do plano de ações convergentes entre diferentes atores. Os roadmaps apresentados contribuem para compreender e comunicar os desafios para estabelecimento desse setor, considerando uma visão de longo prazo e identificando os recursos 
necessários para alcançar os resultados almejados, no intuito de nortear a realização de ações para consolidação do setor e aproveitamento das oportunidades de mercados por parte das empresas brasileiras e, principalmente, do estado de Minas Gerais.

\section{REFERÊNCIAS}

OLIVEIRA, M. G. et al. Roadmapping: uma abordagem estratégica para o gerenciamento da inovação em produtos, serviços e tecnologias. Rio de Janeiro: Elsevier, 2012.

PHAAL, R.; FARRUKH, C.J.P.; PROBERT, D. Roadmapping for strategy and innovation: aligning technology and markets in a dynamic world. University of Cambridge, Institute for Manufacturing, 2010.

PHAAL, R.; FARRUKH, C. J. P.; PROBERT, D. Technology roadmapping: a planning framework for evolution and revolution. Elsevier: Technological Forecasting \& Social Change, 71, p.5-26, 2004.

INTEL. Developing Solutions for the Internet of Things. White Paper. 2014.

JUNIPER RESEARCH. 'Internet of Things' connected devices to almost triple to over 38 billion units by 2020. Disponível em: http://www.juniperresearch.com/press/press- releases/iotconnected-devices-to-triple-to-38-bn-by-2020. Acesso em: 18 de Agosto de 2016.

STAMFORD, C. Gartner`s 2014 Hype Cycle for Emerging Technologies Maps the Journey to Digital Business. GARTNER, 2014. Disponível em: http://www.gartner.com/newsroom/id/2819918., Acesso em: 12 de junho de 2016. 